\title{
Abordagem do Doente com Psoríase pela Medicina Geral e Familiar: Algoritmo de Referenciação e Gestão Partilhada com a Dermatologia
}

\author{
Management of Psoriasis by Family Physicians: Referral \\ Algorithm and Shared Management with Dermatology
}

\author{
Tiago TORRES $\square^{1,2,3}$, Martinha HENRIQUE ${ }^{4}$, Hugo OLIVEIRA ${ }^{5}$, Madalena RODRIGUES ${ }^{6}$, Paulo FERREIRA ${ }^{7}$, \\ Paulo MORAIS ${ }^{8}$, Sérgio ALVES ${ }^{9}$, Tiago Castro LOPES ${ }^{10}$, Rui CERNADAS ${ }^{11}$ \\ Acta Med Port 2021 Oct;34(10):682-689 $\cdot$ https://doi.org/10.20344/amp.13159
}

\section{RESUMO}

Introdução: A implementação de modelos capazes de melhorar a referenciação, por forma a garantir a qualidade e precocidade do diagnóstico, o melhor tratamento e seguimento do doente psoriático é fundamental.

Material e Métodos: Um painel de médicos de Medicina Geral e Familiar e Dermatologia discutiu e criou um algoritmo de referenciação simples, eficaz e célere de doentes com psoríase.

Resultados: O algoritmo proposto inicia-se quando o clínico de Medicina Geral e Familiar suspeita de psoríase. Caso haja dúvidas no diagnóstico, o doente deve ser referenciado para a dermatologia. No caso de um diagnóstico confirmado, compete ao clínico de Medicina Geral e Familiar avaliar a gravidade e perfil de respondedor do doente, rastrear comorbilidades e a possibilidade de artrite psoriática. Se a psoríase for ligeira, deverão ser iniciados tratamentos tópicos, sendo que, se não houver melhoria clínica ou ocorrer agravamento da doença, o doente deverá ser referenciado para a dermatologia. Se a psoríase for considerada moderada a grave, em localizações de elevado impacto, ou em idade pediátrica, o doente deverá ser referenciado para a dermatologia. Para que o seguimento e tratamento destes doentes seja partilhado, é fundamental que o médico de Medicina Geral e Familiar tenha o conhecimento necessário sobre os tratamentos sistémicos que o doente está a fazer e os seus efeitos adversos.

Discussão e Conclusão: Apenas uma gestão partilhada do doente psoriático poderá tornar possível o melhor tratamento e seguimento destes doentes, a utilização mais racional dos recursos médicos disponíveis, proporcionando ao doente a melhor qualidade de vida possível.

Palavras-chave: Dermatologia; Encaminhamento e Consulta; Medicina Geral e Familiar; Psoríase/tratamento

\section{ABSTRACT}

Introduction: The implementation of models capable of improving referral quality, limiting the growth of waiting lists in hospitals, and ensuring the best possible treatment and follow-up of the psoriatic patient is of the utmost importance.

Material and Methods: A panel of Family Physicians and Dermatologists discussed and created a simple and effective algorithm of referral for patients with psoriasis.

Results: The proposed algorithm starts when the Family Physician suspects of psoriasis. In case of diagnostic doubt, the patient should be referred to Dermatology. In case of a confirmed diagnosis, the Family Physician should assess the patient's severity and responder profile, evaluate comorbidities and assess the presence of psoriatic arthritis. If psoriasis is mild, topical treatments should be initiated, and if there is no clinical improvement or worsening of the disease, the patient should be referred to Dermatology. If psoriasis is moderate to severe, is located in high impact locations, or in pediatric age, the patient should be referred to Dermatology. In order to enable shared management in terms of follow-up and treatment of these patients, it is critical that the Family Physician has the necessary knowledge regarding the systemic treatments used in psoriasis and their side effects.

Discussion and Conclusion: Only a shared management of the psoriatic patient can allow for the best treatment and follow-up of these patients, a more rational use of available medical resources, thus giving the patient the best possible quality of life.

Keywords: Dermatology; Family Medicine; General Practice; Psoriasis/therapy; Referral and Consultation

\section{INTRODUÇÃO}

A psoríase é uma doença crónica, sistémica, imuno-mediada, com elevado impacto físico e psicológico. ${ }^{1-3} \mathrm{O}$ seu impacto na qualidade de vida dos doentes é superior

ao de outras patologias consideradas mais graves, como a insuficiência cardíaca e a diabetes, sendo também elevado o impacto na qualidade de vida dos conviventes. ${ }^{3-5}$

1. Departamento de Dermatologia. Centro Hospitalar Universitário do Porto. Porto. Portugal.

2. Instituto de Ciências Biomédicas Abel Salazar. Universidade do Porto. Porto. Portugal.

3. Instituto Médico de Estudos Imunológicos. Porto. Portugal.

4. Serviço de Dermatologia. Centro Hospitalar de Leiria. Leiria. Portugal.

5. Unidade de Fototerapia. Consulta de Fototerapia e Psoríase. Serviço de Dermatologia. Centro Hospitalar e Universitário de Coimbra. Coimbra. Portugal.

6. Unidade de Saúde Familiar Castelo. Agrupamento de Centros de Saúde Arrábida. Sesimbra. Portugal.

7. Unidade de Psoríase. Hospital CUF Descobertas. Lisboa. Portugal.

8. Serviço de Dermatologia. Centro Hospitalar de Trás-os-Montes e Alto Douro. Vila Real. Portugal.

9. Serviço de Dermatologia. Hospital das Forças Armadas. Porto. Portugal.

10. Unidade de Saúde Familiar Serzedelo. Agrupamento de Centros de Saúde Alto Ave. Guimarães. Portugal.

11. Serviços de Saúde Ocupacional. Continental Mabor. Lousado. Portugal.

$\triangle$ Autor correspondente: Tiago Torres. torres.tiago@outlook.com

Recebido: 19 de novembro de 2019 - Aceite: 06 de janeiro de 2020 - First published: 24 de março de 2020 - Online issue published: 01 de outubro de 2021 Copyright @ Ordem dos Médicos 2021 
Estima-se que a psoríase afete $2 \%$ - 3\% da população ${ }^{2} \mathrm{e}$ que em Portugal existam 250000 doentes. ${ }^{6}$ Nos doentes com psoríase grave, ${ }^{7}$ devido à elevada morbilidade, nomeadamente cardiovascular, estima-se que a esperança média de vida esteja diminuída em cerca de cinco anos. ${ }^{4,5,8}$

A gestão partilhada do doente psoriático pelo clínico de Medicina Geral e Familiar (MGF) e o dermatologista é fundamental para a eficácia na gestão da doença. Contudo, apesar do crescente conhecimento sobre a doença e o aumento das opções terapêuticas, continuam a existir lacunas relativamente ao diagnóstico e à otimização do tratamento, pelo que ainda existe uma elevada taxa de subdiagnóstico e sub-tratamento e subdiagnóstico nos doentes com psoríase. ${ }^{9}$

A ausência de componente obrigatório de dermatologia na formação específica de MGF poderá contribuir para esta situação uma vez que os cuidados de saúde primários têm um papel preponderante na abordagem e referenciação destes doentes.

O clínico MGF pode desempenhar um papel importante no diagnóstico da doença, no tratamento das formas ligeiras, na referenciação para o dermatologista dos casos mais graves ${ }^{10}$ e na gestão das comorbilidades e complicações relacionadas com terapêutica sistémica. Para que esta referenciação seja apropriada torna-se fundamental a criação de um algoritmo de referenciação, simples, informativo e eficaz, que possa ser utilizado pelo clínico de MGF na sua prática clínica de rotina. Nos casos mais graves, cabe ao dermatologista informar o médico de família acerca dos tratamentos sistémicos aplicados ao doente, para que este possa ser corretamente co-monitorizado nos cuidados de saúde primários. Assim, a partilha da gestão do doente será o mais eficaz possível por forma a diminuir o impacto da psoríase na morbilidade e mortalidade dos doentes. ${ }^{10}$

\section{MATERIAL E MÉTODOS}

Em março de 2019 reuniu-se um painel de peritos em Medicina Geral e Familiar e dermatologistas com experiência clínica no tratamento de doentes com psoríase. Foi discutida a gestão partilhada do doente psoriático e foi elaborado um algoritmo inicial sobre a referenciação, gestão e tratamento deste tipo de doente. O texto e algoritmo consensual final foram acordados quando o painel se reuniu novamente em maio de 2019. Todos os autores contribuíram e estiveram ativamente envolvidos na preparação deste consenso e respetivo algoritmo.

\section{ALGORITMO DE REFERENCIAÇÃO DO DOENTE PSO- RIÁTICO}

Na Fig. 1 é apresentado um algoritmo de referenciação do doente psoriático, partindo da suspeita de psoríase por parte do clínico de MGF.

\section{Diagnóstico de psoríase}

O diagnóstico de psoríase é essencialmente clínico. Assim, torna-se fundamental conhecer bem as características das diferentes formas de psoríase, por forma a fazer um diagnóstico correto. ${ }^{11}$ Existem diversos fenótipos clínicos que incluem psoríase em placas, gutata, pustulosa, eritrodérmica, palmo-plantar, inversa e ungueal. ${ }^{12} \mathrm{~A}$ psoríase em placas é a mais comum, com cerca de $90 \%$ dos casos. ${ }^{11,13}$

Apesar do diagnóstico de psoríase na maioria dos casos não suscitar dúvidas pelas manifestações clínicas características, é necessário que o médico de família esteja atento a possíveis diagnósticos diferenciais para cada um dos tipos de psoríase. Na Tabela 1 estão resumidas as principais manifestações clínicas de cada tipo de psoríase assim como os possíveis diagnósticos diferenciais. No caso de dúvidas no diagnóstico sugere-se que o doente

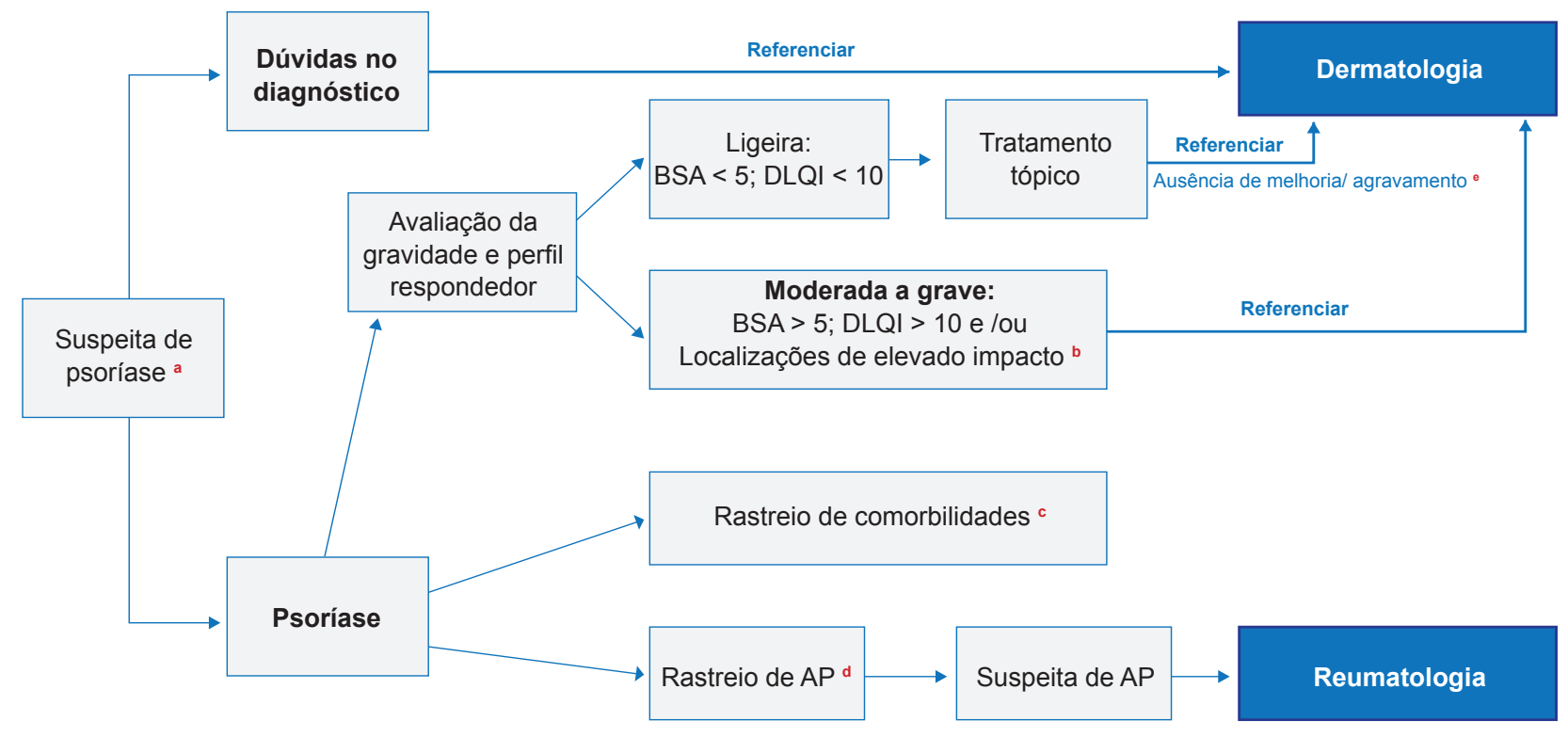

Figura 1 - Algoritmo de referenciação do doente psoriático pela Medicina Geral e Familiar para a Dermatologia

${ }^{a}$ Em caso de doente pediátrico, deverá ser sempre referenciado para a dermatologia; ${ }^{\text {b }}$ Localizações de elevado impacto: couro cabeludo, palmo-plantar, unhas, genital; ${ }^{c}$ Em caso de comorbilidade grave, consultar secção sobre comorbilidades do presente artigo; ${ }^{\mathrm{d} C}$ Consultar secção sobre artrite psoriática do presente artigo; ${ }^{\mathrm{e}}$ Excluindo falta de adesão à terapêutica. BSA: body surface area; DLQI: Dermatology Life Quality Index; AP: artrite psoriática 
seja referenciado para a dermatologia. No caso do doente pediátrico, após suspeita ou confirmação do diagnóstico, independente da gravidade da doença, deverá ser sempre referenciado para a consulta de dermatologia.

\section{Avaliação da gravidade e perfil respondedor}

Após confirmação do diagnóstico pelo clínico de MGF é necessário avaliar a gravidade da doença. A gravidade da doença pode ser avaliada pelo Psoriasis Area and Severity Index (PASI). No entanto, e para além de ser muitas vezes utilizado apenas em contexto de investigação, apresenta também várias desvantagens, sendo muito extenso, complexo, com falta de sensibilidade e reduzida precisão. ${ }^{14} \mathrm{As}-$ sim, opta-se por utilizar o índice Body Surface Area (BSA), mais fácil de utilizar, baseado na percentagem de superfície corporal afetada, na localização das lesões e no impacto na

Tabela 1 - Manifestações clínicas e diagnóstico diferencial dos diferentes tipos de psoríase

\begin{tabular}{|c|c|c|}
\hline Tipo de psoríase & Manifestações clínicas & Diagnóstico diferencial \\
\hline Em placas & $\begin{array}{l}\text { Pápulas e placas eritematosas, bem definidas, recobertas por } \\
\text { escamas brancas prateadas aderentes; } \\
\text { Distribuição habitualmente simétrica, no couro cabeludo, } \\
\text { cotovelos, joelhos e região lombar, podendo localizar-se em } \\
\text { qualquer parte do corpo; } \\
\text { Prurido que pode resultar no desenvolvimento de } \\
\text { escoriações. }{ }^{35-37}\end{array}$ & $\begin{array}{l}\text { Micose fungóide; } \\
\text { Dermatofitia; } \\
\text { Dermatite seborreica. }^{38}\end{array}$ \\
\hline Gutata & $\begin{array}{l}\text { Aparecimento súbito de lesões características de psoríase, } \\
\text { arredondadas com < } 1 \mathrm{~cm} \text { no tronco e extremidades; } \\
\text { Lesões psoriáticas semelhantes a gotas, pequenas, dispersas, } \\
\text { monomórficas e no mesmo estado de evolução; } \\
\text { Afeta frequentemente crianças e adolescentes após uma } \\
\text { infeção por estreptococos ou do trato respiratório superior; } \\
\text { progride para psoríase em placas em alguns indivíduos. }{ }^{39-41}\end{array}$ & $\begin{array}{l}\text { Pitiríase liquenóide crónica; } \\
\text { Sífilis secundária; } \\
\text { Papulose linfomatóide; } \\
\text { Pitiríase rósea de Gilbert. }{ }^{38}\end{array}$ \\
\hline Pustulosa & $\begin{array}{l}\text { Múltiplas pústulas estéreis, dolorosas, com base subjacente } \\
\text { eritematosa; } \\
\text { A forma generalizada é rara e requer hospitalização; } \\
\text { A forma palmo-plantar é caracterizada por pústulas estéreis, } \\
\text { amarelas-castanhas nos pés e nas mãos. } .^{42-44}\end{array}$ & $\begin{array}{l}\text { Pustulose exantemática aguda generalizada; } \\
\text { Doença de Sneddon-Wilkinson; } \\
\text { Doença de Reiter; } \\
\text { Foliculite; } \\
\text { Foliculite eosinofílica. }{ }^{44}\end{array}$ \\
\hline Eritrodérmica & $\begin{array}{l}\text { Eritema generalizado envolvendo }>80 \% \text { da superfície corporal; } \\
\text { Pode ocorrer devido a suspensão repentina de medicação } \\
\text { (p. ex. corticosteróides), reação medicamentosa ou infeção } \\
\text { sistémica. }{ }^{45-47}\end{array}$ & $\begin{array}{l}\text { Toxicodermia; } \\
\text { Dermatite atópica; } \\
\text { Dermatite de contacto; } \\
\text { Síndrome de Sezary; } \\
\text { Pitiríase rubra pilar. }{ }^{11}\end{array}$ \\
\hline Palmo-plantar & $\begin{array}{l}\text { Eritema, escamas finas e fissuras nas palmas das mãos e nas } \\
\text { plantas dos pés. } .^{48-50}\end{array}$ & $\begin{array}{l}\text { Eczema disidrótico; } \\
\text { Dermatite de contacto; } \\
\text { Pitiríase rubra pilar; } \\
\text { Queratodermia palmo-plantar adquirida; } \\
\text { Dermatofitia. }{ }^{49}\end{array}$ \\
\hline Inversa & $\begin{array}{l}\text { Placas eritematosas, pouco descamativas, localizadas nas } \\
\text { superfícies flexoras e nas pregas da pele como zona perianal, } \\
\text { infra mamária, axilas, inguinal e infra glúteos. }{ }^{11,51}\end{array}$ & $\begin{array}{l}\text { Intertrigo bacteriano; } \\
\text { Intertrigo causado por Candida; } \\
\text { Dermatite seborreica; } \\
\text { Eritrasma; } \\
\text { Infeção fúngica; } \\
\text { Dermatite de contacto. }{ }^{38,52}\end{array}$ \\
\hline Ungueal & $\begin{array}{l}\text { Afeta } 80 \% \text { dos doentes com psoríase; } \\
\text { Onicólise, picotado ungueal, "gotas de óleo" de descoloração } \\
\text { amarela ou salmão, hiperqueratose ungueal, hemorragias } \\
\text { punctiformes, eritema da lúnula; } \\
\text { Distrofia. }{ }^{11,32,53}\end{array}$ & $\begin{array}{l}\text { Onicomicoses; } \\
\text { Distrofia ungueal; } \\
\text { Síndrome ungueal de marcha assimétrica. }{ }^{51,53}\end{array}$ \\
\hline Genital & $\begin{array}{l}\text { Todos os grupos etários; } \\
\text { Cerca de } 1 / 3 \text { dos doentes com psoríase sofre de psoríase } \\
\text { genital; } \\
\text { Placas eritematosas bem demarcadas, brilhantes, finas, sem } \\
\text { descamação. }{ }^{54,55}\end{array}$ & $\begin{array}{l}\text { Doenças sexualmente transmissíveis; }{ }^{55} \\
\text { Intertrigo bacteriano; } \\
\text { Intertrigo causado por Candida spp; } \\
\text { Dermatite seborreica; } \\
\text { Eritrasma; } \\
\text { Infeção fúngica; } \\
\text { Dermatite de contacto. }{ }^{38,52}\end{array}$ \\
\hline
\end{tabular}


qualidade de vida. Para este último deverá ser utilizado o questionário Dermatology Quality of Life Index (DLQI).

O BSA é estimado com base na percentagem de área do corpo afetada, sendo que $1 \%$ corresponde à área da palma do doente. ${ }^{14}$ Desta forma, se BSA $<3 \%$ a psoríase é considerada ligeira, entre $3 \%$ - $10 \%$ moderada e superior a $10 \%$ é considerada grave. ${ }^{15}$

No doente adulto, o DLQI é o mais conhecido entre os questionários de qualidade de vida associado a patologias dermatológicas, estando validado para a população portuguesa. ${ }^{16} \mathrm{O}$ DLQI demonstra elevada validade, fiabilidade e consistência interna. Para além disso, tem a vantagem de ser um questionário curto, contém apenas 10 itens, e é fácil de preencher pelos doentes. A escala varia entre 0 e 30, em que o valor mínimo 0 - 1 corresponde a nenhum impacto na qualidade de vida e o valor máximo entre 21 - 30 corresponde a um extremo impacto na qualidade de vida ${ }^{17}$

Assim, por opinião do painel de peritos, ficou preconizado que a gravidade da doença seja avaliada pelo médico de família com base nos índices BSA e DLQI e que a psoríase deve ser considerada moderada a grave se BSA $>5$ e/ou DLQI > 10. ${ }^{10}$ Nos doentes em que os valores dos índices sejam inferiores, a psoríase deverá ser classificada como ligeira.

Nos casos em que a psoríase for categorizada como ligeira (BSA < 5; DLQI < 10), devem ser iniciados tratamentos tópicos pelo clínico de MGF, sendo a primeira avaliação feita entre 4 - 6 semanas após o início da terapêutica para verificar adesão e resposta inicial à terapêutica. A segunda visita deverá ser feita após três meses, seguida de visitas anuais. ${ }^{10} \mathrm{O}$ doente deve ser alertado para, caso haja agravamento, agendar nova consulta. ${ }^{18}$ Se ocorrer ausência de melhoria, após confirmação de adesão à terapêutica, ou agravamento dos sintomas por um período de 2 - 3 meses, o doente deve ser referenciado para a dermatologia. ${ }^{10}$
Os doentes com psoríase moderada a grave devem ser referenciados para a dermatologia por forma a iniciar fototerapia ou terapêutica sistémica.

Se as lesões psoriáticas surgirem em localizações de elevado impacto, como face, palmo-plantar, unhas, couro cabeludo ou genitais, a psoríase deve ser considerada moderada a grave mesmo que a área afetada seja pequena. Estas lesões têm marcada repercussão na atividade física, saúde emocional e capacidade para efetuar as atividades do quotidiano do doente. ${ }^{19}$ Desta forma estes doentes devem ser referenciados para a dermatologia.

A estratégia terapêutica deverá passar pela resolução das lesões evitando recidivas ou agravamento. ${ }^{16}$ É importante referir que com os recentes avanços nas opções terapêuticas é possível, de forma realística, ter como objetivo terapêutico a resolução completa ou quase completa das lesões. ${ }^{20}$

\section{Terapêutica tópica}

É opinião do painel que a terapêutica tópica deve ser utilizada em doentes com psoríase ligeira. Na psoríase moderada a grave os agentes tópicos são muitas vezes utilizados como adjuvantes da terapêutica sistémica. ${ }^{16}$ Contudo, a satisfação e adesão à terapêutica tópica é muitas vezes baixa devido a dificuldade e tempo de aplicação, em parte devido à sua cosmeticidade. ${ }^{21-23}$ Assim, as diferentes opções terapêuticas, sumarizadas na Tabela 2, devem ser discutidas entre o médico de MGF e o doente por forma a otimizar a terapêutica. ${ }^{24}$

\section{GESTÃO PARTILHADA DO DOENTE PSORIÁTICO}

\section{Terapêutica sistémica}

Os tratamentos tópicos incluídos na Tabela 2 são, na maioria dos casos, insuficientes para controlar a psoríase moderada a grave pelo que, uma vez referenciado para a

Tabela 2 - Tratamento tópico da psoríase no adulto ${ }^{56-58}$

\begin{tabular}{lll}
\hline Localização & Tratamento & Formulação \\
\hline Couro cabeludo & $\begin{array}{l}\text { Corticosteróide de potência alta } \\
\text { Queratolíticos + corticosteróide de potência alta } \\
\text { Calcipotriol + betametasona }\end{array}$ & $\begin{array}{l}\text { Loção, creme, champô } \\
\text { Loção } \\
\text { Gel }\end{array}$ \\
Face & $\begin{array}{l}\text { Corticosteróide de potência baixa/ média } \\
\text { Inibidores da calcineurina (off label) }\end{array}$ & Creme \\
Pregas & $\begin{array}{l}\text { Corticosteróide de potência média } \\
\text { Inibidores da calcineurina (off label) }\end{array}$ & Cremada \\
Tronco e membros & $\begin{array}{l}\text { Calcipotriol + betametasona } \\
\text { Derivados da Vitamina } D(\text { calcitriol, tacalcitol) }\end{array}$ & Creme, pomada \\
Palmas e plantas & $\begin{array}{l}\text { Corticosteróide de potência alta } \\
\text { Queratolíticos }\end{array}$ & Espuma**, gel, pomada \\
& Calcipotriol + betametasona & Pomada \\
Genital & $\begin{array}{l}\text { Corticosteróide de potência baixa/ média } \\
\text { Inibidores da calcineurina (off label) }\end{array}$ & Pomada \\
\end{tabular}

* Não utilizar em áreas extensas, nem a longo prazo; ** Fase de indução: 1 x dia, máximo 4 semanas, máximo 15 g/dia; após fase de indução 2 - 3 x semana; *** melhores resultados 
dermatologia, é necessário que o doente inicie fototerapia ou terapêutica sistémica, sendo os agentes tópicos utilizados como adjuvantes em lesões localizadas. ${ }^{16}$

A partir deste momento inicia-se uma gestão partilhada do doente psoriático pelos médicos de família e dermatologistas que deve ser traduzida numa estreita e continuada comunicação para que a terapêutica da psoríase seja o mais eficaz possível.

A fototerapia com radiação UVB ou psoraleno com radiação UVA (PUVA) pode ser utilizada como alternativa à terapia tópica ou como adjuvante desta. Apesar de a fototerapia ser realizada em unidades de fototerapia e requerer, por parte do doente, tempo disponível para as sessões, esta é temporariamente eficaz para alguns doentes e não está associada aos comprovados efeitos adversos dos imunossupressores sistémicos. Contudo, algumas modalidades de fototerapia têm sido associadas ao aumento de neoplasias malignas cutâneas, sendo contra-indicadas em doentes de elevado risco. ${ }^{18}$

As terapêuticas sistémicas convencionais (metotrexato, ciclosporina e acitretina) estão associadas a toxicidade em diferentes órgãos, especialmente a longo prazo, necessitando de monitorização clínica e analítica periódica. Adicionalmente são várias as contra-indicações e interações medicamentosas a ter em conta quando se inicia o tratamento.

No últimos anos, decorrente dos avanços no conhecimento acerca dos mecanismos fisiopatológicos da psoríase, foram desenvolvidas novas terapêuticas denominadas de agentes biológicos, ${ }^{16}$ que mostraram ser mais eficazes e seguras do que terapêuticas sistémicas convencionais, além de permitirem tratamentos a longo prazo e não terem interações medicamentosas. Na Tabela 3 estão incluídos os tratamentos sistémicos disponíveis para a psoríase assim como os efeitos adversos mais frequentes. Embora a monitorização destes efeitos adversos deva ser feita pelo dermatologista, é importante que o especialista em MGF os conheça, no caso de observação do doente ou do seguimento longitudinal do mesmo. Em relação aos fármacos biológicos há alguns pontos comuns a todos que devem ser tidos em conta pelo médico de família ${ }^{16,25}$ :

- Infeções

- rastreio de tuberculose e vigilância nos doentes com prova de tuberculina negativa eteste IGRAnegativo ou indeterminado;

- Vacinação

- as vacinas vivas estão contraindicadas em doentes sob terapêutica biológica. No caso de necessidade, é fundamental a discussão com o dermatologista.

- as vacinas da gripe e pneumocócica devem ser recomendadas, assim como a atualização do Plano Nacional de Vacinação.

- Gravidez

- a terapia biológica está contraindicada. Neoplasias

- devem ser incentivados os programas de rastreios, conforme preconizado para a população em geral;

- a terapia biológica está contraindicada em doentes com neoplasia há menos de 5 anos;

- deve ser avaliado o risco de cancro cutâneo não-melanoma.

- Cirurgias

- a terapia biológica deve ser suspendida em cirurgias programadas tipo III (cirurgias contaminadas, ou seja as realizadas em tecidos colonizados por flora microbiana abundante, de difícil descontaminação, na ausência de processo infecioso local. Consideram-se contaminadas as cirurgias realizadas no

Tabela 3 - Tratamentos sistémicos utilizados na psoríase e seus efeitos adversos

\begin{tabular}{|c|c|}
\hline Fármaco & Efeitos adversos comuns \\
\hline \multicolumn{2}{|l|}{ Agentes sistémicos orais } \\
\hline Metotrexato 59 & $\begin{array}{l}\text { Estomatite, dispepsia, náusea, perda de apetite, dor abdominal, alterações da } \\
\text { função hepática }\end{array}$ \\
\hline Ciclosporina ${ }^{60}$ & Hiperlipidémia, tremor, cefaleias, hipertensão, hirsutismo, disfunção renal. \\
\hline Acitretina ${ }^{61}$ & $\begin{array}{l}\text { Secura da mucosa ocular, (conjuntivite, xeroftalmia), visão turva, diminuição de } \\
\text { visão noturna, queratite ulcerativa, epistaxis, rinite, xerostomia, sede, queilite, } \\
\text { prurido, alopecia, esfoliação da pele, alterações da função hepática, hiperlipidémia. }\end{array}$ \\
\hline \multicolumn{2}{|c|}{ Efeitos adversos mais comuns a ter em atenção } \\
\hline $\begin{array}{l}\text { Anti-TNF- } \alpha \\
\text { Etanercept, }^{62} \text { Adalimumab }^{63} \text { Infliximab } \\
\end{array}$ & Reativação de tuberculose-risco superior com anti TNF- $\alpha$ \\
\hline $\begin{array}{l}\text { Anti IL-12/23 } \\
\text { Ustecinumab }\end{array}$ & Sintomas de doença desmielinizante - essencialmente com anti TNF- $\alpha$ \\
\hline $\begin{array}{l}\text { Anti IL-17 } \\
\text { Secucinumab, }{ }^{66} \text { Ixecizumab, }{ }^{67} \text { Brodalumab }^{68}\end{array}$ & $\begin{array}{l}\text { Candidíase - essencialmente com anti IL-17 } \\
\text { Infeções - apresentação menos típica e potencialmente mais grave }\end{array}$ \\
\hline $\begin{array}{l}\text { Anti IL-23 } \\
\text { Guselcumab, }{ }^{69} \text { Tildracizumab, } \\
\text { Risancizumab }\end{array}$ & Reação no local da injecção \\
\hline
\end{tabular}


cólon, reto e ânus; em tecido com lesões cruentas e cirurgias de traumatismo crânio encefálicos abertos) ou IV (cirurgias infetadas, ou seja, as realizadas em qualquer tecido, na presença de processo infecioso local).

\section{Comorbilidades}

O doente com psoríase tem um risco aumentado para diversas comorbilidades e o risco aumenta com a gravidade da doença. ${ }^{26}$ A gestão e abordagem das comorbilidades deverão ser partilhadas entre o clínico de MGF e o dermatologista. Assim, a gestão destes doentes passa por tratar a doença como um todo e não apenas focada nas manifestações dermatológicas. ${ }^{12}$ As comorbilidades mais comuns associadas à psoríase são ${ }^{26}$.

- Doenças cardiometabólicas: diabetes, obesidade, dislipidémia, hipertensão;

- Doença cardiovascular: acidente vascular cerebral, enfarte do miocárdio;

- Doença inflamatória intestinal;

- Esteatose hepática não-alcoólica;

- Ansiedade e depressão.

A avaliação do doente psoriático pelo médico de família deve incluir a história clínica completa e deve ser feito um exame físico objetivo por forma a rastrear as comorbilidades mais comuns. Todos os doentes psoriáticos, independentemente da gravidade, têm um risco elevado de depressão, pelo que é recomendável o rastreio regular desta patologia. Para isso, uma questão simples como "Quanto é que a psoríase o afeta?" é o suficiente para iniciar o diálogo e avaliar o impacto psicológico causado pela psoríase. ${ }^{18}$ É opinião do painel que o rastreio das comorbilidades associadas à psoríase deve ser semestral para doentes em tratamento sistémico e anual para doentes em tratamento tópico. ${ }^{27,28}$

Para além das comorbilidades é importante referir alguns hábitos de consumo, como o tabaco, que tem um efeito negativo na psoríase e na síndrome metabólica, e o álcool, cujo consumo é mais comum em doentes com psoríase. ${ }^{29,30}$

\section{Artrite psoriática}

A artrite psoriática é uma artropatia inflamatória crónica de envolvimento articular periférico e/ou axial, bem como de tecidos moles. ${ }^{31}$

A associação entre o envolvimento ungueal e o desenvolvimento de artrite psoriática é elevada, sendo que cerca de $70 \%$ dos doentes com artrite psoriática apresentam psoríase ungueal. ${ }^{32}$ Desta forma, o envolvimento ungueal deve ser considerado como preditor para o desenvolvimento futuro de artrite psoriática.

O diagnóstico da artrite psoriática é, na maioria dos casos, um desafio, e atrasos no diagnóstico podem conduzir a um pior prognóstico. Cerca de $80 \%$ dos casos desenvolve-se após o aparecimento das lesões cutâneas de psoríase, ${ }^{26}$ pelo que o clínico de MGF e o dermatologista têm ambos um papel crucial na identificação precoce de artrite psoriática. Em 2015 foi desenvolvido um questionário para dermatologistas mas que pode ser aplicado à MGF, que pode ajudar a identificar os doentes com queixas articulares que devem ser referenciados à reumatologia ${ }^{33,34} \mathrm{e}$, nas unidades hospitalares sem esta valência, à medicina interna:

- Acorda durante a noite por dor nas articulações?

- Sofre de rigidez matinal nas articulações?

- Alguma vez teve alguma articulação inchada?

- Alguma vez teve dor e inchaço da totalidade de algum dedo (dedo em forma de salsicha)?

Este simples questionário, juntamente com a avaliação ungueal, apresenta elevada sensibilidade na deteção de artrite psoriática: se três ou mais respostas forem positivas e se houver envolvimento ungueal, a sensibilidade é de $86,9 \%$ e a especificidade de $71,3 \%$ para o diagnóstico de artrite psoriática, nos doentes com psoríase da pele. ${ }^{33}$

É opinião do painel que a avaliação das unhas e o rastreio de artrite psoriática seja feito anualmente pelo médico de família e, em caso de positividade para as questões acima, o doente seja referenciado para a consulta de reumatologia. Nas unidades hospitalares sem esta valência, o doente deve ser referenciado para a consulta de medicina interna.

\section{DISCUSSÃO E CONCLUSÃO}

O papel do especialista em MGF é fundamental na abordagem do doente com psoríase, por forma a diminuir o sub-diagnóstico, o sub-tratamento e a sub-referenciação. É também fundamental o seu papel no tratamento correcto dos casos ligeiros, mitigando a referenciação desnecessária para o dermatologista, mantendo os cuidados no médico de família. Cabe também ao clínico de MGF referenciar ao dermatologista os casos suspeitos de psoríase em idade pediátrica, assim como, em estreita colaboração com a dermatologia, diagnosticar precocemente a artrite psoriática.

No início do tratamento da psoríase é importante comunicar ao doente os objetivos do tratamento e o nível de compromisso necessário para a obtenção de uma resolução completa ou quase completa das lesões cutâneas. Apesar de a terapêutica sistémica ser iniciada pelo dermatologista, é importante que o médico de família tenha conhecimento da mesma para que possa acompanhar e vigiar o doente da melhor forma. Assim, para além de em todas as visitas o clínico de MGF dever questionar o doente acerca da evolução da doença e terapêutica, também o dermatologista tem o dever de informar o clínico de MGF do plano terapêutico para que este possa monitorizar os possíveis efeitos adversos.

Igualmente importante é a promoção de estilos de vida saudáveis que permitirão um melhor controlo das comorbilidades, assim como o melhor controlo da doença. Mais uma vez, o papel do médico de família revela-se fundamental na gestão partilhada das comorbilidades.

Em conclusão, a relação e a comunicação entre as duas especialidades, MGF e dermatologia, permitirá a colocação 
do doente no centro dos cuidados e uma visão partilhada e interdisciplinar do doente e da doença.

\section{CONTRIBUTO DOS AUTORES}

TT, MH: Elaboração e revisão crítica.

HO, MR, PF, PM, SA: Elaboração do artigo.

TCL, RC: Revisão crítica do manuscrito.

\section{CONFLITOS DE INTERESSE}

O autor Tiago Torres declara ter recebido honorários referentes a consultoria ou por ter sido palestrante em eventos patrocinados pela AbbVie, Amgen, Arena Pharmaceuticals, Boehringer Ingelheim, Biogen, Celgene, Bristol-Myers
Squibb, Biocad, Janssen, LEO-Pharma, Eli-Lilly, MSD, Novartis, Pfizer, Samsung Bioepis e Sanofi. Todos os outros autores declaram ter recebido honorários por participar em Advisory Boards da Novartis.

\section{FONTES DE FINANCIAMENTO}

A Novartis Portugal financiou as reuniões e o acesso a toda a bibliografia necessária. A assistência editorial foi proporcionada por Irina Duarte PhD, da X2-Science Solutions. Esta assistência foi financiada pela Novartis Portugal. A Novartis Portugal não teve qualquer papel na elaboração do manuscrito ou na decisão de submeter o manuscrito para publicação.

\section{REFERÊNCIAS}

1. Johnson-Huang LM, Lowes MA, Krueger JG. Putting together the psoriasis puzzle: an update on developing targeted therapies. Dis Model Mech. 2012;5:423-33.

2. Di Meglio P, Villanova F, Nestle FO. Psoriasis. Cold Spring Harb Perspect Med. 4:a015354.

3. Nestle FO, Kaplan DH, Barker J. Psoriasis. N Engl J Med. 2009;361:496509.

4. Rapp SR, Feldman SR, Exum ML, Fleischer AB Jr, Reboussin DM Psoriasis causes as much disability as other major medical diseases. J Am Acad Dermatol. 1999;41:401-7.

5. Abuabara K, Azfar RS, Shin DB, Neimann AL, Troxel AB, Gelfand JM Cause-specific mortality in patients with severe psoriasis: a populationbased cohort study in the U.K. Br J Dermatol. 2010;163:586-92.

6. Torres T, Filipe P, Selores M. Impact of biosimilars in psoriasis treatment. Acta Med Port. 2013;26:646-8.

7. Armstrong EJ, Harskamp CT, Armstrong AW. Psoriasis and major adverse cardiovascular events: a systematic review and meta-analysis of observational studies. J Am Heart Assoc. 2013;2:e000062.

8. Hu SC, Lan CC. Psoriasis and cardiovascular comorbidities: focusing on severe vascular events, cardiovascular risk factors and implications for treatment. Int J Mol Sci. 2017;18:2211.

9. Lebwohl MG, Bachelez H, Barker J, Girolomoni G, Kavanaugh A, Langley RG, et al. Patient perspectives in the management of psoriasis: results from the population-based Multinational Assessment of Psoriasis and Psoriatic Arthritis Survey. J Am Acad Dermatol. 2014;70:871-81.

10. Bilal J, Malik SU, Riaz IB, Kurtzman DJ. Psoriasis and psoriatic spectrum disease: a primer for the primary care physician. Am J Med. 2018;131:1146-54.

11. Raychaudhuri SK, Maverakis E, Raychaudhuri SP. Diagnosis and classification of psoriasis. Autoimmun Rev. 2014;13:490-5.

12. Schadler ED, Ortel B, Mehlis SL. Biologics for the primary care physician: review and treatment of psoriasis. Dis Mon. 2019;65:51-90.

13. Levine D, Gottlieb A. Evaluation and management of psoriasis: an internist's guide. Med Clin North Am. 2009;93:1291-303.

14. Bozek $A$, Reich $A$. The reliability of three psoriasis assessment tools: psoriasis area and severity index, body surface area and physician global assessment. Adv Clin Exp Med. 2017;26:851-6.

15. Horn EJ, Fox KM, Patel V, Chiou CF, Dann F, Lebwohl M. Are patients with psoriasis undertreated? Results of National Psoriasis Foundation survey. J Am Acad Dermatol. 2007;57:957-62

16. Marques Pinto G, Filipe P. Normas de boa prática para o tratamento da psoríase em placas em idade não pediátrica com biológicos. Acta Med Port. 2012;25:125-41.

17. Finlay AY, Khan GK. Dermatology Life Quality Index (DLQI)--a simple practical measure for routine clinical use. Clin Exp Dermatol. 1994;19:210-6

18. Young $M$, Aldredge L, Parker P. Psoriasis for the primary care practitioner. J Am Assoc Nurse Pract. 2017;29:157-78.

19. Menter A, Korman NJ, Elmets CA, Feldman SR, Gelfand JM, Gordon KB et al. Guidelines of care for the management of psoriasis and psoriatic arthritis: section 6. Guidelines of care for the treatment of psoriasis and psoriatic arthritis: case-based presentations and evidence-based conclusions. J Am Acad Dermatol. 2011:65:137-74.

20. Torres T, Puig L. Treatment goals for psoriasis: should PASI 90 become the standard of care? Actas Dermosifiliogr. 2015;106:155-7.

21. Devaux S, Castela A, Archier E, Gallini A, Joly P, Misery L, et al. Adherence to topical treatment in psoriasis: a systematic literature review. J Eur Acad Dermatol Venereol. 2012;26:61-7.

22. Duffin KC, Yeung $H$, Takeshita J, Krueger GG, Robertson AD, Troxel AB et al. Patient satisfaction with treatments for moderate-to-severe plaque psoriasis in clinical practice. Br J Dermatol. 2014;170:672-80.

23. Wu JJ, Lynde CW, Kleyn CE, Iversen L, van der Walt JM, Carvalho A, et al. Identification of key research needs for topical therapy treatment of psoriasis - a consensus paper by the International Psoriasis Council. J Eur Acad Dermatol Venereol. 2016;30:1115-9

24. Torsekar R, Gautam MM. Topical therapies in psoriasis. Indian Dermato Online J. 2017;8:235-45.

25. Smith $\mathrm{CH}$, Jabbar-Lopez ZK, Yiu ZZ, Bale T, Burden AD, Coates LC, et al. British Association of Dermatologists guidelines for biologic therapy for psoriasis 2017. Br J Dermatol. 2017;177:628-36.

26. Armstrong AW, Schupp C, Bebo B. Psoriasis comorbidities: results from the National Psoriasis Foundation surveys 2003 to 2011. Dermatology. 2012;225:121-6.

27. Elmets CA, Leonardi CL, Davis DM, Gelfand JM, Lichten J, Mehta $\mathrm{NN}$, et al. Joint AAD-NPF guidelines of care for the management and treatment of psoriasis with awareness and attention to comorbidities. J Am Acad Dermatol. 2019;80:1073-113.

28. Dauden E, Blasco AJ, Bonanad C, Botella R, Carrascosa JM, GonzálezParra E, et al. Position statement for the management of comorbidities in psoriasis. J Eur Acad Dermatol Venereol. 2018;32:2058-73.

29. Naldi L. Psoriasis and smoking: links and risks. Psoriasis. 2016;6:65-71.

30. Naldi L, Peli L, Parazzini F. Association of early-stage psoriasis with smoking and male alcohol consumption: evidence from an Italian casecontrol study. Arch Dermatol. 1999;135:1479-84.

31. Raposo I, Torres T. Nail psoriasis as a predictor of the development of psoriatic arthritis. Actas Dermosifiliogr. 2015;106:452-7.

32. Oram $Y$, Akkaya AD. Treatment of nail psoriasis: common concepts and new trends. Dermatol Res Pract. 2013;2013:180496.

33. Garg N, Truong B, Ku JH, Devere TS, Ehst BD, Blauvelt A, et al. A novel, short, and simple screening questionnaire can suggest presence of psoriatic arthritis in psoriasis patients in a dermatology clinic. Clin Rheumatol. 2015;34:1745-51.

34. Villani AP, Rouzaud M, Sevrain M, Barnetche T, Paul C, Richard MA, et al. Symptoms dermatologists should look for in daily practice to improve detection of psoriatic arthritis in psoriasis patients: an expert group consensus. J Eur Acad Dermatol Venereol. 2014;28:S27-32.

35. Griffiths CE, Barker JN. Pathogenesis and clinical features of psoriasis Lancet. 2007;370:263-71.

36. Langley RG, Krueger GG, Griffiths CE. Psoriasis: epidemiology, clinica features, and quality of life. Ann Rheum Dis. 2005;64:ii18-25.

37. Menter A, Gottlieb A, Feldman SR, Van Voorhees AS, Leonardi CL, Gordon KB, et al. Guidelines of care for the management of psoriasis and psoriatic arthritis: section 1. Overview of psoriasis and guidelines of care for the treatment of psoriasis with biologics. J Am Acad Dermatol. 2008;58:826-50

38. Lisi P. Differential diagnosis of psoriasis. Reumatismo. 2007:59:S56-60

39. Krishnamurthy K, Walker A, Gropper CA, Hoffman C. To treat or not to treat? Management of guttate psoriasis and pityriasis rosea in patients with evidence of group A Streptococcal infection. J Drugs Dermatol. 2010;9:241-50. 
40. Naldi L, Peli L, Parazzini F, Carrel CF. Family history of psoriasis, stressful life events, and recent infectious disease are risk factors for a first episode of acute guttate psoriasis: results of a case-control study. J Am Acad Dermatol. 2001;44:433-8.

41. Martin BA, Chalmers RJ, Telfer NR. How great is the risk of further psoriasis following a single episode of acute guttate psoriasis? Arch Dermatol. 1996;132:717-8

42. Baker H, Ryan TJ. Generalized pustular psoriasis. A clinical and epidemiological study of 104 cases. Br J Dermatol. 1968;80:771-93.

43. Ohkawara A, Yasuda $H$, Kobayashi $H$, Inaba $Y$, Ogawa $H$, Hashimoto I, et al. Generalized pustular psoriasis in Japan: two distinct groups formed by differences in symptoms and genetic background. Acta Derm Venereol. 1996;76:68-71.

44. Mengesha YM, Bennett ML. Pustular skin disorders: diagnosis and treatment. Am J Clin Dermatol. 2002;3:389-400.

45. Egeberg A, Thyssen JP, Gislason GH, Skov L. Prognosis after hospitalization for erythroderma. Acta Derm Venereol. 2016;96:959-62.

46. Farber EM, Nall L. Erythrodermic (exfoliative) psoriasis. Cutis. 1993:51:79-82.

47. Balasubramaniam P, Berth-Jones J. Erythroderma: $90 \%$ skin failure. Hosp Med. 2004;65:100-2.

48. Janagond AB, Kanwar AJ, Handa S. Efficacy and safety of systemic methotrexate vs. acitretin in psoriasis patients with significant palmoplantar involvement: a prospective, randomized study. J Eur Acad Dermatol Venereol. 2013;27:e384-9.

49. Engin B, Askin O, Tuzun Y. Palmoplantar psoriasis. Clin Dermatol. 2017;35:19-27.

50. Raposo I, Torres T. Palmoplantar psoriasis and palmoplantar pustulosis: current treatment and future prospects. Am J Clin Dermatol. 2016;17:349-58.

51. Syed ZU, Khachemoune A. Inverse psoriasis: case presentation and review. Am J Clin Dermatol. 2011;12:143-6.

52. Ladizinski B, Lee KC, Wilmer E, Alavi A, Mistry N, Sibbald RG. A review of the clinical variants and the management of psoriasis. Adv Skin Wound Care. 2013;26:271-84.

53. Salomon J, Szepietowski JC, Proniewicz A. Psoriatic nails: a prospective clinical study. J Cutan Med Surg. 2003;7:317-21.

54. Czuczwar P, Stepniak A, Goren A, Wrona W, Paszkowski T, Pawlaczyk $M$, et al. Genital psoriasis: a hidden multidisciplinary problem - a review of literature. Ginekol Pol. 2016;87:717-21.

55. Meeuwis KA, de Hullu JA, de Jager ME, Massuger LF, van de Kerkhof PC, van Rossum MM. Genital psoriasis: a questionnaire-based survey on a concealed skin disease in the Netherlands. J Eur Acad Dermatol Venereol. 2010;24:1425-30.

56. Chiricozzi A, Pimpinelli N, Ricceri F, Bagnoni G, Bartoli L, Bellini M, et al. Treatment of psoriasis with topical agents: recommendations from a Tuscany Consensus. Dermatol Ther. 2017;30.

57. Dattola A, Silvestri M, Bennardo L, Del Duca E, Longo C, Bianchi L, et al. Update of calcineurin inhibitors to treat inverse psoriasis: a systematic review. Dermatol Ther. 2018;31:e12728.

58. Torres T, Filipe P. New calcipotriol/betamethasone dipropionate foam formulation for the treatment of psoriasis: an overview. Journal of the Portuguese Society of Dermatology and Venereology. 2018;76:12.
59. Resumo das Características do Medicamento. Metotrexato. Aprovado pela Agência Europeia de Medicamentos 1990. [consultado 2020 jan 04]. Disponível em: https://www.ema.europa.eu/en/documents/productinformation/nordimet-epar-product-information_pt.pdf.

60. Resumo das Características do Medicamento. Ciclosporina. Aprovado pela Agência Europeia de Medicamentos 1990. [consultado 2020 jan 04]. Disponível em: http://app7.infarmed.pt/infomed/download_ficheiro. php?med_id=7745\&tipo_doc=rcm

61. Resumo das Características do Medicamento. Acitretina. Aprovado pela Agência Europeia de Medicamentos 1990. [consultado 2020 jan 04]. Disponível em: http://app7.infarmed.pt/infomed/download_ficheiro. php?med id=6012\&tipo_doc $=\mathrm{rcm}$

62. Resumo das Características do Medicamento. Etanercept. Aprovado pela Agência Europeia de Medicamentos 2000. [consultado 2020 jan 04]. Disponível em: https://www.ema.europa.eu/en/documents/productinformation/enbrel-epar-product-information_pt.pdf.

63. Resumo das Características do Medicamento. Adalimumab. Aprovado pela Agência Europeia de Medicamentos 2008. [consultado 2020 jan 04]. Disponível em: https://www.ema.europa.eu/en/documents/productinformation/humira-epar-product-information_pt.pdf.

64. Resumo das Características do Medicamento. Infliximab. Aprovado pela Agência Europeia de Medicamentos 1999. [consultado 2020 jan 04]. Disponível em: https://www.ema.europa.eu/en/documents/productinformation/remicade-epar-product-information_pt.pdf.

65. Resumo das Características do Medicamento. Ustekinumab. Aprovado pela Agência Europeia de Medicamentos 2009. [consultado 2020 jan 04]. Disponível em: https://www.ema.europa.eu/en/documents/productinformation/stelara-epar-product-information_pt.pdf.

66. Resumo das Características do Medicamento. Secukinumab. Aprovado pela Agência Europeia de Medicamentos 2015. [consultado 2020 jan 04]. Disponível em: https://www.ema.europa.eu/en/documents/productinformation/cosentyx-epar-product-information pt.pdf.

67. Resumo das Características do Medicamento. Ixekizumab. Aprovado pela Agência Europeia de Medicamentos 2016. [consultado 2020 jan 04]. Disponível em: https://www.ema.europa.eu/en/documents/productinformation/taltz-epar-product-information_pt.pdf.

68. Resumo das Características do Medicamento. Brodalumab. Aprovado pela Agência Europeia de Medicamentos 2017. [consultado 2020 jan 04]. Disponível em: https://www.ema.europa.eu/en/documents/productinformation/kyntheum-epar-product-information_pt.pdf.

69. Resumo das Características do Medicamento. Guselkumab. Aprovado pela Agência Europeia de Medicamentos 2017. [consultado 2020 jan 04]. Disponível em: https://www.ema.europa.eu/en/documents/productinformation/tremfya-epar-product-information_pt.pdf.

70. Resumo das Características do Medicamento. Tildrakizumab. Aprovado pela Agência Europeia de Medicamentos 2018. [consultado 2020 jan 04]. Disponível em: https://www.ema.europa.eu/en/documents/productinformation/ilumetri-epar-product-information_pt.pdf.

71. Krueger JG, Ferris LK, Menter A, Wagner F, White A, Visvanathan S et al. Anti-IL-23A mAb BI 655066 for treatment of moderate-to-severe psoriasis: Safety, efficacy, pharmacokinetics, and biomarker results of a single-rising-dose, randomized, double-blind, placebo-controlled trial. J Allergy Clin Immunol. 2015;136:116-24. 